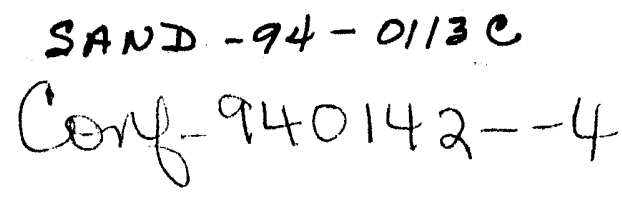

\title{
Computational Methods for Describing the Laser-Induced Mechanical Response of Tissue
}

\author{
Timothy Trucano, J. Michael McGlaun, and Archie Farnsworth
}

Sandia National Laboratories

P. O. Box 5800

Albuquerque, New Mexico 87185-0819

\begin{abstract}
Detailed computational modeling of laser surgery requires treatment of the photoablation of human tissue by high intensity pulses of laser light and the subsequent thermomechanical response of the tissue. Three distinct physical regimes must be considered to accomplish this: (1) the immediate absorption of the laser pulse by the tissue and following tissue ablation, which is dependent upon tissue light absorption characteristics; (2) the near field thermal and mechanical response of the tissue to this laser pulse; and (3) the potential far field (and longer time) mechanical response of witness tissue. Both (2) and (3) are dependent upon accurate constitutive descriptions of the tissue. We will briefly review tissue absorptivity and mechanical behavior, with an emphasis on dynamic loads characteristic of the photoablation process. In this paper our focus will center on the requirements of numerical modeling and the uncertainties of mechanical tissue behavior under photoablation. We will also discuss potential contributions that computational simulations can make in the design of surgical protocols which utilize lasers, for example, in assessing the potential for collateral mechanical damage by laser pulses.
\end{abstract}

\section{INTRODUCTION}

Energetic (i.e. non-diagnostic) laser-tissue interactions have been studied in a variety of contexts for potential and actual clinical usage (Konov, et $\mathrm{al}^{10}$ and Sona ${ }^{20}$. Applications of interest include the following: (1) opthamology, where lasers can be used to perform microsurgery and for which there is interest in the use of lasers for retinal "sculpting"; (2) soft tissue surgery, including scar tissue removal and tumor removal; (3) angioplasty, in which soft or hard plaque is penetrated and removed within blood vessels, such as the aorta; and (4) endoscopic (laser light delivered via fiber optic systems) surgeries of various kinds, in particular for breakup of kidney and gall stones. In addition, lasers continue to be investigated for employment in bone surgery. 
The laser-tissue interaction that takes place in all of these treatments is dominated by the evaporation and removal of tissue due to approximately constant volume deposition of laser energy within the tissue.

A wide variety of laser systems and pulse characteristics have been studied experimentally ${ }^{20}$. The illumination conditions vary from $\mathrm{CO}_{2}$ lasers operated in $\mathrm{CW}$ format to excimer lasers having nanosecond pulse widths. The range of intensities (power per unit area) produced by lasers of medical interest can vary from less than $10^{5}$ to more than $10^{10} \mathrm{~W} / \mathrm{cm}^{2}$. Complicating the actual interaction of a particular laser pulse with a particular tissue is the fact that the pulse may be delivered via fiber optic systems, which considerably modify the dynamic response of the tissue to the deposited energy.

The greatest therapeutic concem in regard to laser surgical techniques, beyond the actual ability of the proposed laser to remove the required tissue, is the control of collateral damage to neighboring healthy tissue. The main reason for surgical interest in lasers is the extraordinary precision offered by their use. Unfortunately, a major side effect of the thermal removal of tissue by laser light - photoablation - is thermal and mechanical damage to neighboring healthy tissue. Incidental thermal damage has been the subject of a great deal of research, both experimental and analytical, over the past decade (see, for example, Muller, et $\mathrm{al}^{13}$, Döschel and Müller ${ }^{5}$, Sagi, et $\mathrm{al}^{17}$, Walsh, et $\mathrm{al}^{24}$ ). We will briefly review the major issues developed in this work in Section 2 below.

An additional concern, especially when harder tissue is the object of the laser interaction or when higher intensity laser pulses are used, is potential ancillary mechanical effects and residual mechanical damage. There has been considerably less discussion of the etiology of mechanical effects in tissue in the literature on medical laser applications. Nonetheless, there is a body of experimental data that suggests that the mechanical response of tissue to laser pulse interactions is important (Walsh, et al ${ }^{24}$, Teng, et $\mathrm{al}^{22}$, and Zweig and Weber ${ }^{26}$, to name three references). Therefore, we should try to understand the relevant mechanical phenomena. Examination of the $\mathrm{r}$ : echanical consequences of the laser interaction is less amenable to simplified analytical approaches for several reasons. First, tissue mechanical response is tightly coupled to the delivery of the laser energy, making static approximations, that are somewhat effective in thermal modeling, problematic for this application. Second, the dynamic loading undergone by the tissue due to photoablation is nonlinear. Third, tissue mechanical behavior under dynamic loads is generally nonlinear. (See Dingus and Shafer ${ }^{4}$ for an introductory exposition of nonlinear dynamic loading resulting 
from laser-tissue interactions.) Fourth, the important mechanical consequences of the laser interaction are often multidimensional.

It is the purpose of this paper to discuss the origin and modeling of tissue mechanical response to laser pulse interactions. Our discussion will be mainly qualitative. After reviewing aspects of the laser-tissue interaction in Section 2 , we will turn our attention to requirements for simulation of these interactions using large scale computation. In Section 3 we will review laser pulse absorption, aspects of dynamic loading phenomena, relevant constitutive behavior of tissue, and general computational approaches for simulating tissue behavior. Solid dynamics computer codes that are coupled to laser energy sources can be used to model the laser-tissue interactions in the breadth that we discuss. Our presentation summarizes the inputs that would be required to utilize these tools for effective modeling of the laser-tissue interaction in general and of proposed surgical protocols in particular.

\section{LASER ILUUMINATION AND TISSUE RESPONSE}

We will focus our discussion on the use of pulsed lasers. The "ideal" laser-tissue interaction is depicted schematically in Figure 1. There, we have sketched the absorption of an instantaneous, perfect spatially rectangular laser pulse, having fluence (energy per area) sufficient to evaporate the absorption volume of tissue. It is assumed that the absorption is loss-free, so that all of the energy of the pulse is absorbed by the tissue. In addition, it is also depicted in this figure that the evaporated material is instantaneously ejected in a recoil-free manner from the surrounding tissue, leaving a void with no thermally excited region beyond its boundary and with no residual mechanical loads existing in the surrounding tissue. A series of such laser pulses would produce a "perfect" incision and no collateral tissue damage. The geometry of Figure 1 is also effectively one dimensional.

In virtually ALL respects this picture is incomplete. First, laser pulses are not instantaneous, nor are they perfectly rectangular spatially. More realistic temporal and spatial pulse distributions have also been drawn in Figure 1 (the dashed curves). The temporal spread of the pulse (often approximately Gaussian in form) means that tissue is changing its thermodynamic state during the absorption of the laser pulse. Also, the finite time for delivery of the pulse energy implies that part of the energy can also be lost from the absorption region into neighboring tissues via thermal conduction. Neighboring tissues are then susceptible to thermal damage. A simple criterion based on the characteristic time associated with thermal conduction, the so-called thermal 
relaxation time, can be used to determine pulse widths necessary to minimize thermal damage. Walsh, et al ${ }^{24}$ estimate thermal relaxation times in tissue to be on the order of one microsecond or more for characteristic absorption coefficients, based on the following formula:

$$
\tau_{R}=\frac{(1 / \alpha)^{2}}{4 x}
$$

In (1), $\tau_{R}$ is the thermal relaxation time, $\alpha$ is a suitable light absorption coefficient for tissue, and $x$ is a thermal diffusivity for tissue. $\left(\alpha-10,000 \mathrm{~cm}^{-1}\right.$, suitable for 0.25 micron light, and $\mathrm{x}-1.3 \times 10^{-3}\left(\mathrm{~cm}^{2} / \mathrm{s}\right)$ were used in the thermal relaxation time estimate given above.) The inference from the thermal relaxation time estimate is that usable medical laser pulses for ablative surgical applications should be on the order of one microsecond or less to minimize thermal damage to ancillary tissue.

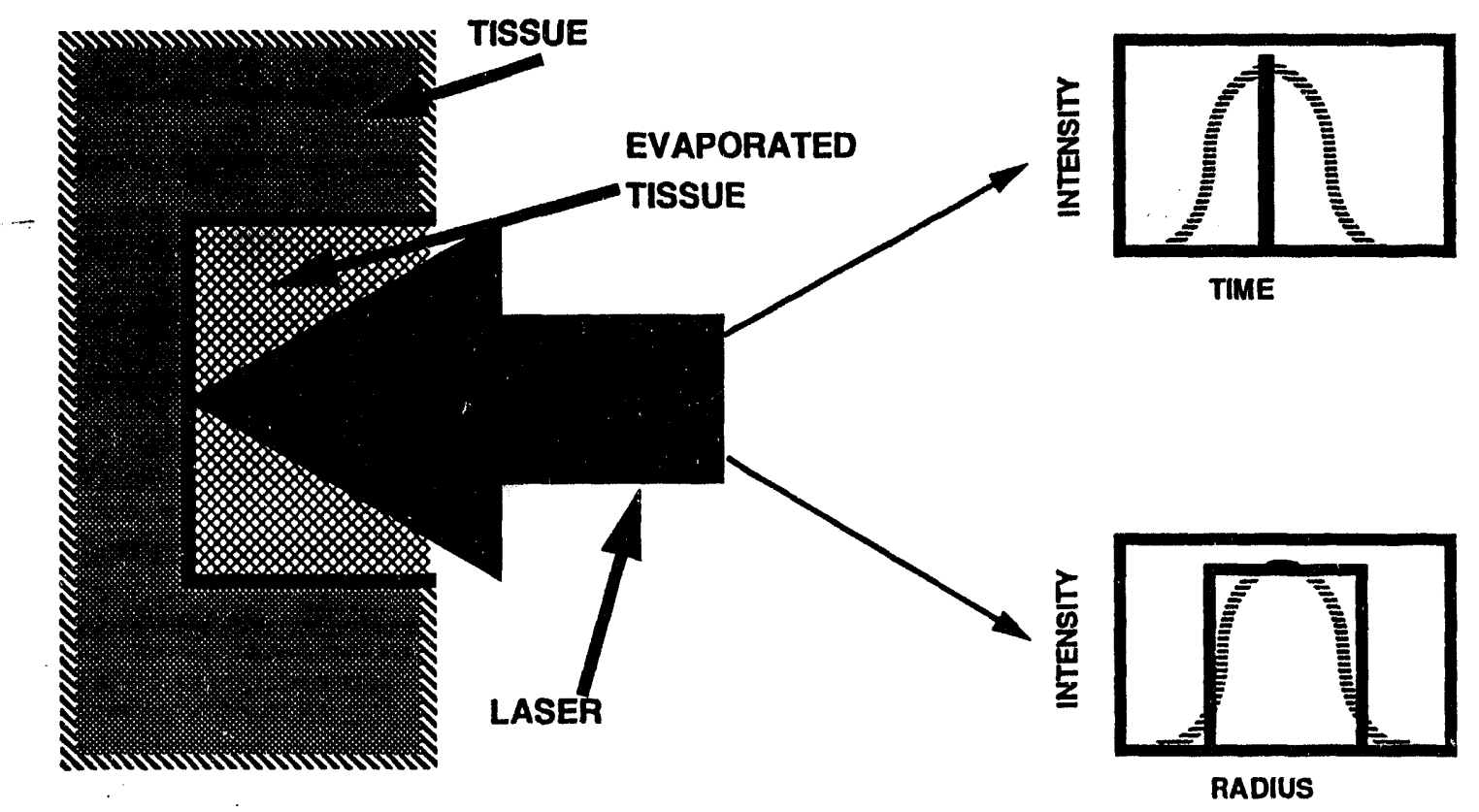

Figure 1. A "perfect" laser-tissue interaction.

The realistic spatial distribution of the pulse often has far more energy in the radial "wings" of the pulse than we have sketched in Figure 1 . This means that the spatial boundary of the absorbed region is not precisely defined. Rather, a certain amount of 
tissue will be heated but will NOT evaporate, at least on the same time sci le as of the central portion of the illuminated region. The spatial broadening of real laser pulses is of considerable therapeutic concern, because this decreases the accuracy of the energy deposition, and increases the potential healthy tissue damage due to thermal effects (Sagi, et $\mathrm{al}^{17}$ ).

Second, even for the "ideal" laser pulse, the absorption would not be perfect. Light absorption in tissue, a complex heterogeneous material, is a very complicated process. Physical parameters which influence the absorption characteristics will vary during the laser pulse. Only a fraction of the laser pulse (typically a large fraction!) will actually be absorbed by a macroscopic volume of tissue, leading to eventual tissue ablation. The absorption characteristics of the next pulse will also be modified by the behavior of the tissue under the proceeding pulse.

Third, the response of the tissue to the absorbed energy is not instantaneous evaporation without recoil. The details of the thermodynamic transformation of cool tissue to a vapor depend upon the temporal characteristics of the laser pulse, the wavelength of the light, the energy contained in the pulse, and fine details of the tissue composition. The phenomenon of tissue ablation is a threshold effect, in which a minimum energy per unit volume must be absorbed from the laser pulse before any tissue ablation can occur. In soft tissue this threshold energy is approximately the heat of vaporization of water, from 0.6 to 1.5 $\mathrm{J} / \mathrm{cm}^{2}$. Because of the spatial distribution of the laser pulse, some of the absorbing tissue will not evaporate due to the failure to absorb the required threshold energy. The complete evaporation of these regions will thus require further laser pulses. Most of these details can be empirically combined into experimentally observed ablation rates, which can be expressed as volume of tissue removed per laser pulse, the mass of material removed per pulse, or the number of pulses required to achieve a fixed excavation depth. As an illustration, Walsh and Deutsch ${ }^{23}$ provide a nice set of data for Er.YAG laser illumination of skin, aorta, and bone expressed in terms of depth of tissue removed per pulse. Yet the details are necessary for a full understanding of the evaporation process.

The vaporized tissue is initially at a high pressure because it is heated at approximately constant volume. The high pressure will propagate into the cooler surrounding tissue and the vapor will flow back toward the laser. The mechanical impulse that is delivered by this process is a complex function of the tissue constitutive properties and the laser pulse parameters. It is governed by the conservation of momentum. Simple approxinations can clarify the scaling of delivered impulse versus laser pulse characteristics (see Dingus and Shafer ${ }^{4}$ for a discussion of this impulse). In particular, the pressure induced by the "blow-off" 
of the evaporated tissue, called the ablation pressure, increases with increasing pulse intensity. This fact implies that given constant pulse energy and spot size, as the temporal width of the pulse decreases the ablation pressure increases. In principle, our "ideal" laser pulse would induce a very high ablation pressure. Thus, the only way that the cooler tissue could not move under the impetus of this pressure would be if it was exceptionally rigid.

Because of non-zero ablation pressures and finite tissue strength, the neighboring tissue will indeed recoil under the ablative loads. The implications of this recoil, or more generally, the mechanical response of the tissue, have not been investigated in as much detail as the thermal response of this tissue. There is considerable evidence that the effect is important. For example, mechanical coupling is known to be important in effectively breaking up gall and kidney stones using laser pulses. Detailed data concerning this coupling is presented in Teng, et $a^{22}$. Features of the mechanical coupling are also important in assessing the use of lasers in angioplasty (Furzikov ${ }^{8}$, Srinivasan ${ }^{21}$, Welch, et $\mathrm{al}^{25}$, and Singleton, et $\mathrm{al}^{18}$ ) and in optimizing the delivered laser pulses. A considerable problem is posed by the mechanical response of "soft" and "hard" (calcified) plaque in this procedure. The laser technique which is suitable for ablating and penetrating a soft plaque deposit can produce dangerous fragmentation of hard plaque which could subsequently enter the bloodstream. Since it is difficult to recognize the changing character of plaque during an in vivo laser procedure, and thus when the danger of plaque fragmentation might be present, more detailed analysis of laser-plaque interactions would be a significant advance. Finally, we point out that Walsh, et $\mathrm{al}^{24}$ comment that unlike the laser ablation of soft tissue and bone, which tends to leave circular ablation lesions, the residual ablation lesions for laser-aorta interactions are highly elliptical. The long axis of these elliptical lesions (ablation craters) are observed to be parallel to the circumferential direction of the aorta tissue used in the reported experiments. Aorta is a fiber composite material in which the orientation of the component elastin fibers is circumferential $\left(\mathrm{Fung}^{7}\right)$. Walsh, et $\mathrm{al}^{24}$ suggest that a percentage of the volume of the lesion actually represents tissue tearing in response to the ablation pressures of the laser interaction, which is a purely residual mechanical effect. One would assume that similar phenomena are important in other tissue structures, perhaps near bone joints or muscle attachment points.

In addition, Teng, et $\mathrm{al}^{22}$ also quantified the influence of confining liquid in improving the breaking of stones by laser pulses. In this technique, photoablation pressures are uses to fragment stones. The compressive stress waves that are generated in the body of the stone reflect from inhomegeities and the boundaries of the stone to form states of tension. (This process is discussed in further detail in Section 3.2 below.) These tensile states can then fracture the stone material if the tensile strength of the stone 
is exceeded. The magnitude of the tension produced in the stone increases with the ablation pressure. Teng, et al show that the presence of confining liquid can dramatically increase the ablation pressure delivered to stones by the mechanical effects of laser pulse interactions. In Fabbro, et $\mathbf{a l}^{6}$, a more general treatment of laser-target interactions, the authors show that confinement of the ablation products produced by laser pulses can increase ablation pressures by as much as a factor of ten. Delivery of laser pulses by fiber optics also modify the nature of the confirement of the ablation products. Authors (such as Srinivasan $\mathrm{et}^{\mathrm{a}^{21}}$ ) have reported damage to fiber tips at laser pulse intensities which are known to be below optical breakdown thresholds in the fiber material, typically quartz. This damage could easily be caused by the pressures of the expanding ablative gases created by the laser. Detailed analysis is needed to determine modifications of techniques that would allow greater flexibility in the use of fibers.

To summarize this discussion, we now refer to Figure 2. There, we have depicted a more "realistic" analog of Figure 1. The major features now represented in Figure 2 include an irregular ablated region of tissue, and collateral damaged tissue (either thermal, mechanical, or both). There is also the blown off vapor or plasma that the laser pulse interacts with in delivering the latter portion of its energy to cooler tissue. Finally, we have drawn confining fluid (it could also be solid tissue) and a fiber delivery system, with a propagating stress wave at some distance from the ablation region in the cooler tissue. The implicit geometry is now at least two dimensional (axisymmetry assumed). In fact, the geometry will most likely be three dimensional, but there is little probability that three-dimensional problems will be important for computational studies in the near future. We have also neglected to indicate the heterogeneous nature of the tissue, which complicates laser absorption mechanisms, analysis of thermal damage, and the propagation of the mechanical disturbance.

\section{DISCLAIMER}

This report was prepared as an account of work sponsored by an agency of the United States Government. Neither the United States Government nor any agency thereof, nor any of their employees, makes any warranty, express or implied, or assumes any legal liability or responsibility for the accuracy, completeness, or usefulness of any information, apparatus, product, or process disclosed, or represents that its use would not infringe privately owned rights. Reference herein to any specific commercial product, process, or service by trade name, trademark, manufacturer, or otherwise does not necessarily constitute or imply its endorsement, recommendation, or favoring by the United States Government or any agency thereof. The views and opinions of authors expressed herein do not necessarily state or reflect those of the United States Government or any agency thereof. 


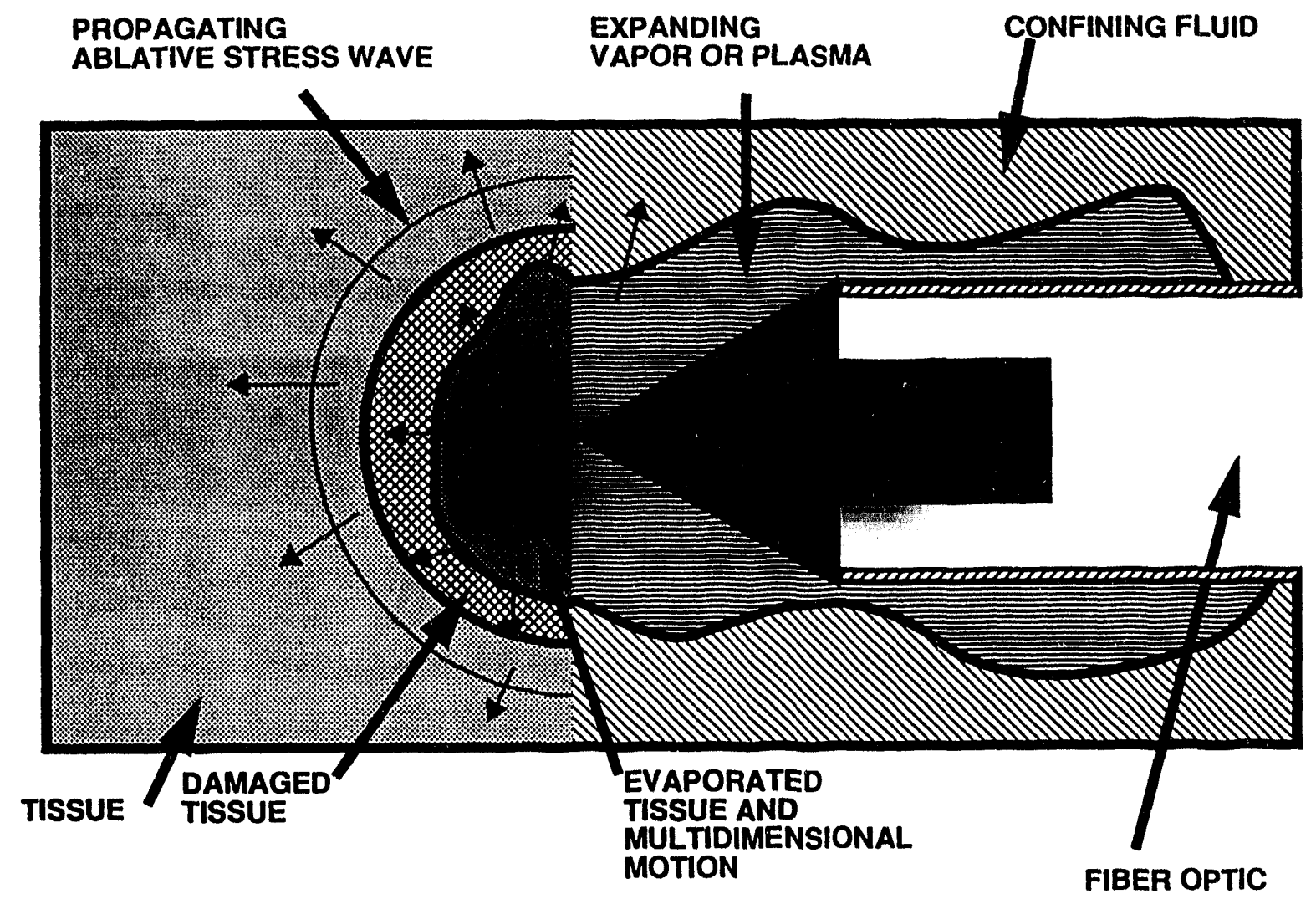

Figure 2. "Realistic" depiction of laser-tissue interaction, during the delivery of a single laser pulse.

Only parts of this picture are subject to analytic or simplified numerical study. However, current computational tools, both hardware and software, offer useful tools for more general analysis of this problem. We will now discuss component aspects of the simulation of this problem.

\section{Synthesis of the Laser-Tissue Interaction via Large-Scale Computation}

The ability to simulate the interaction of medical lasers with human tissues currently exists in several software platforms. A discussion of this capability should include a description of the capabilities of the software, as well as its current limitations. Space does not allow us to touch upon these issues in more than a cursory fashion. Instead, we will include sufficient references to allow the interested reader to pursue the subject in greater depth. 
In Figure 3, we display a conceptual flow chart of the computational analysis of the problem depicted in Figure 2.

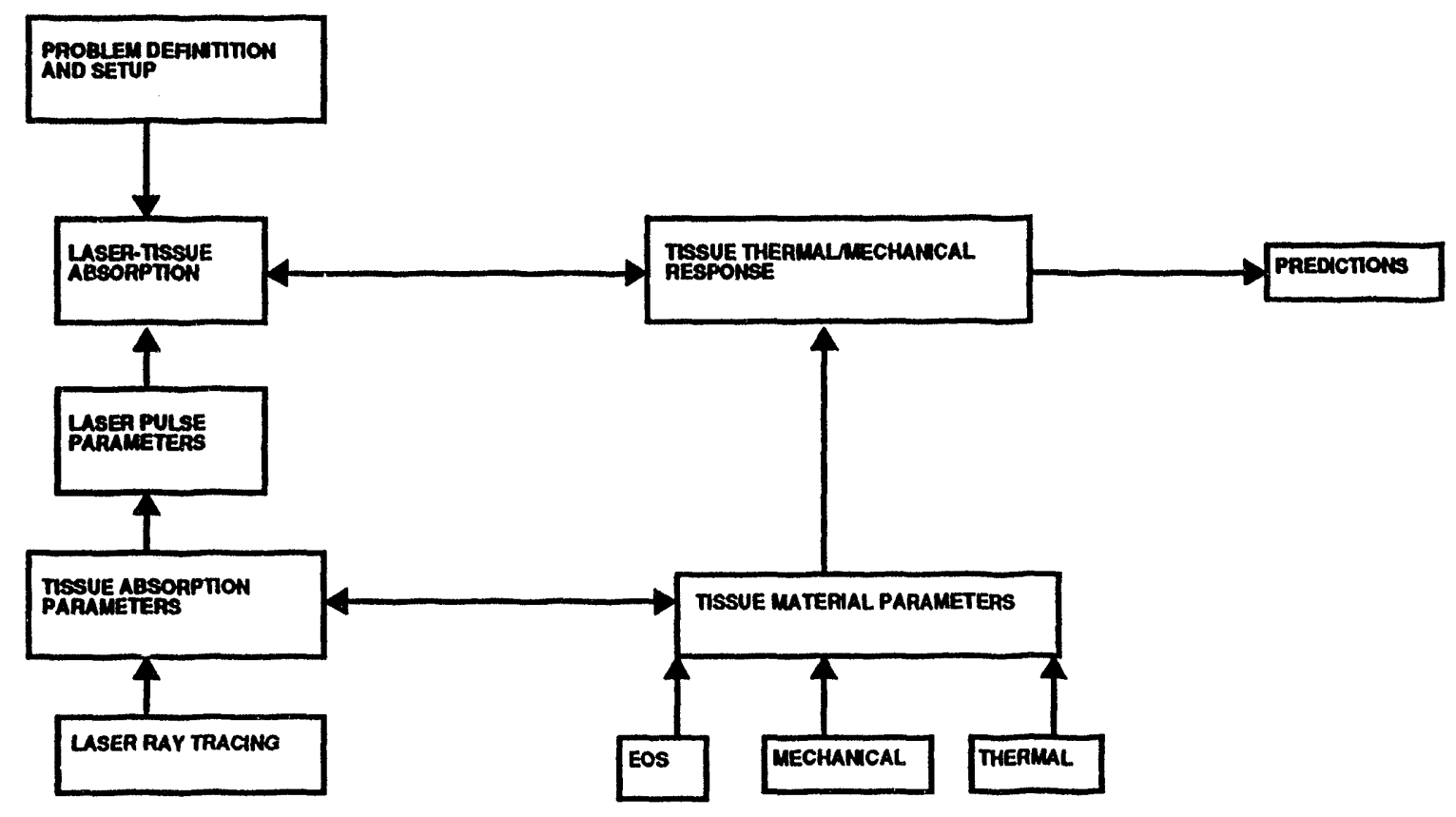

Figure 3. Flow diagram of multi-dimensional analysis of laser-tissue interactions.

Because of the fairly large amount of published analysis of the thermal aspects of the laser-tissue interaction, our discussion will be oriented specifically towards the mechanical interaction phenomenology.

\subsection{Tissue Absorption of Laser Light}

There are three aspects to treating laser light absorption in tissue. First, one must consider the nature of the laser light pulse that impinges on the tissue. Second, tissue absorption characteristics must be modeled. Third, because of the spatial and temporal distribution of the typical pulse of medical interest, ray tracing techniques should be applied to perform the most accurate calculation of pulse propagation in the tissue medium.

The light absorptivity of human tissue is complex in its microscopic aspects, due both to tissue heterogeneities and to the importance of scattering $\left(\right.$ Profio $^{15}$ ). Tissue heterogeneities are sometimes random in nature, so that light propagation in this 
medium must sometimes also be analyzed in terms of propagation in random media. For this paper, we will view tissue as a simple absorber, with the absorption coefficient modified so as to represent an average over the complex detailed behavior. This approach is similar to many other studies and is quite adequate for studies of tissue mechanical response. For additional discussion of tissue light absorption, a relevant reference is Sliney and Wolbarsht ${ }^{19}$.

The absorption coefficient dominates laser pulse absorption at very early times, independent of the intensity of the laser pulse. The absorption coefficient is generally related to the tissue dielectric behavior. The simplest law of direct light absorption in tissue is the Beer law (Sliney and Wolbarsh $\mathrm{t}^{19}$ and Müller, et $\mathrm{al}^{13}$ ) of exponential attenuation. For a one-dimensional, isotropic, and homogeneous medium this law is simply expressed by:

$$
\Phi(x)=\Phi_{0} e^{-\alpha x},
$$

where $\Phi_{0}$ is the incident intensity at $x=0$, and $\Phi(x)$ is the transmitted intensity at $x$. Once again, we stress that all of the microscopic details of the process of light propagation in tissue are contained in the linear absorption coefficient $\alpha$. This parameter is highly dependent on the wavelength of the incident light and on the type and pigmentation of the tissue. Additionally, $\alpha$ will depend upon position for general three-dimensional media that are non-isotropic and heterogeneous. Also, in the form that (2) has been written, light scattering in the tissue has been included in $\alpha$. In fact, this means that even for the assumption of purely one-dimensional isotropic and homogeneous skin, $\alpha$ will actually depend upon $x$ because of the forward peaking of light scattering in tissue. Another physical parameter that influences $\alpha$ is the water content of the tissue. This fact is often used to take advantage of the absorption peaks of water in selecting laser light wavelengths for specific applications. Sliney and Wolbarsht ${ }^{19}$ report values of $\alpha$ that vary from approximately 70 to $300 \mathrm{~cm}^{-1}$ for pale skin, for both deeper and superficial layers, over a light wavelength range of 0.2 to 2.6 microns. Müller, et al $^{13}$ report values varying from approximately 1 to $9000 \mathrm{~cm}^{-1}$ over a similar range of light wavelengths.

For low enough intensities, the tissue light absorption via an exponential law such as (2) dominates the entire laser pulse absorption process. The evaporated tissue is transparent, or almost transparent to the laser light, and direct absorption of light by cool tissue continues for the duration of the pulse and for subsequent pulses. But, if pulse intensities are sufficient to ionize the evaporated tissue (somewhere in the neighborhood of $10^{7} \mathrm{~W} / \mathrm{cm}^{2}$ ), then the mechanism of classical inverse bremsstrahlung 
$\left(\mathrm{Kruer}^{11}\right.$ ) dominates light absorption after formation of the plasma. This completely transforms the absorption process, for the plasma is not transparent to the laser light. In this case, laser energy is no longer directly absorbed by cool tissue. Instead, the light is absorbed in the plasma, with subsequent thermal energy transfer to the cool tissue producing further ablation. The pulse then tends to decouple thermally from the tissue and the resulting thermal transfer to the surrounding tissue is smaller than without plasma formation.

Mechanical coupling effects may become far more prominent with plasma formation because ablation pressures increase in this regime due to the higher laser pulse intensities. There is also evidence that for a range of laser intensities plasma formation may reduce the mechanical coupling. For example, Walsh and Deutsch ${ }^{23}$ have observed decreases in the efficiency of the ablation process in bone when plasma formation occurs. These authors interpreted their data as representing a loss in the pulse energy absorbed by the cool tissue. But, we note that it may also represent the fact that the pulse has decoupled mechanically from the tissue in some way, and this can indirectly influence ablation efficiency. A detailed analysis is required to fully understand this data. Nishioka and Anderson ${ }^{14}$ also observed dependence of stone fracturing efficiency as a function of laser wavelength at intensities for which plasma formed. They found that the breakup of stones was more efficient for shorter light wavelengths. The mechanical coupling in inverse bremsstrahlung is known to be wavelength dependent, with stronger coupling occurring at shorter wavelengths. This is exactly the trend observed by Nishioka and Anderson ${ }^{14}$. We refer the reader to the references for additional details, and simply note that this absorption mechanism influences the thermal and mechanical aspects of tissue ablation.

In addition to accounting for the light absorption mechanisms in tissue, for high accuracy simulations we must also account for the finite temporal and spatial width of laser pulses in computations and the fact that the tissue medium will move during the course of the laser pulse. It is common in the laser-matter interaction community to account for these feature by performing geometric ray tracing to absorb the pulse energy. Accurate ray tracing is particularly important in the regime of plasma formation because of distributed absorption and refraction effects in the plasma. Because ray tracing is a computational technicality, we will not discuss it further here. The techniques are quite straight forward, especially for lasers of the relatively low intensities required for medical applications. A useful reference is Rinker and Bohannon ${ }^{16}$. 


\subsection{Brief Overview of Stress Pulse Phenomena}

This section provides the reader with a brief overview of stress pulse phenomena. The mechanical behavior of materials is concerned with stress wave propagation, distortion, and fragmentation. In the case considered in this paper, the high pressure vaporized tissue will generate a pressure pulse that propagates into the surrounding tissue. The resulting shock wave can heat or damage neighboring tissue. As a result, hard tissue like plaque and bone can fracture where the shocks reflect off material interfaces.

Pressure pulses change shape as they propagate. This occurs because a material's sound speed is not a constant. The sound speed increases as the pressure increases. The higher pressure portions of a pulse travel faster than the lower pressure portions. This causes the front of a pulse to steepen until it changes into a discontinuity, as shown in Figure 4. This discontinuous pressure pulse is called a shock wave.

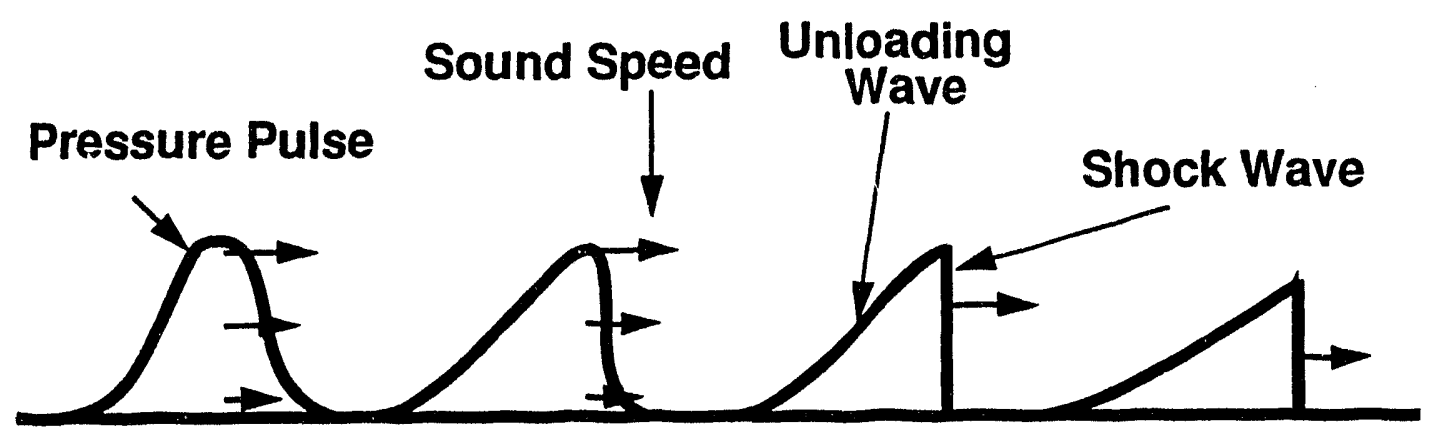

Figure 4. Compression Pulse Builds to Shock Wave

An unloading wave follows the shock, dropping the pressure to ambient values across the unloading wave. The pressure dependent sound speed also causes the unloading wave to change shape. The lower pressure portion of the pulse travels slower than the high pressure portion, so the unloading wave spreads out. However, the unloading wave velocity is also greater than the velocity of the shock front itself. This causes attenuation of the shock pressure as the unloading wave overtakes the shock front. This effect is important for laser-tissue interactions. Due to the short duration of the laser pulse, attenuation of the shock will lower the amplitude. 
Some of the pressure pulse's kinetic energy is also irreversibly dissipated into internal energy across the shock wave because of the dissipative nature of the tissue. This heats the material, so that it is at an elevated temperature after unloading. Because of the kinetic energy removed from the shock wave by dissipation in the tissue, the wave loses energy and the peak pressure attenuates as the wave propagates due to this mechanism as well.

A solid can support tensile stress until it reaches a value called the tensile strength where it fractures. A compression pulse can fracture a material when the pulse reflects from a material interface. For simplicity, we will discuss reflection from a free surface. In this case, the fracture occurs inside the body and is referred to as a spall. The reason this happens is that a compression pulse reflects from a free surface as a mirror image tensile pulse. The reflected pulse has the same shape as the incident pulse, except that the left-hand and right-hand sides are interchanged and it is tensile rather than compressive. 'The reflected tensile pulse and the incoming compression pulse add together to give a complex pulse shape as shown in Figure 5. The region of highest tensile stress occurs inside the body at a distance from the free surface, as shown in Figure 5. As the amplitude of the stress pulse increases, the amplitude of the reflected tensile pulse also increases. For a high enough compression pulse, the tensile pulse exceeds the tensile strength of the material and a spall occurs.

1

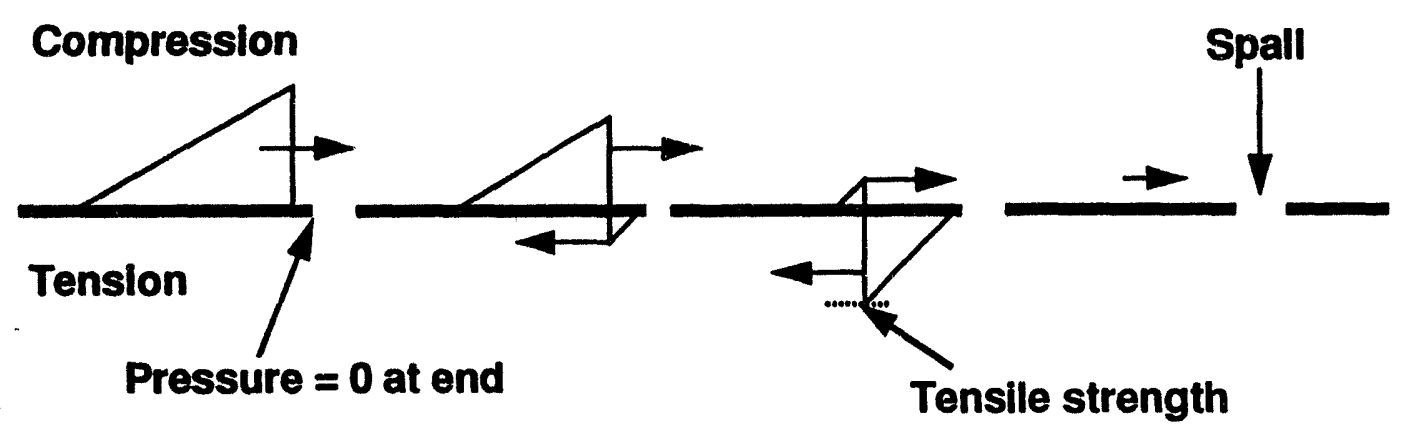

Figure 5. Reflected compression pulse and spall

Measured values of material tensile strength are highly sensitive to the loading process, in particular to the shape and strength of the shock in a spall process. The tensile strength is generally an increasing function of the loading strain rates. Thus, tensile strengths measured in static tests will be smaller than spall strengths inferred from wave propagation experiments. 
More complex stress pulses interacting with irregular internal structures can generate numerous small fractures inside a material, as shown in Figure 6. The fractures may coalesce into long, irregular cracks. Alternatively, the fractures may not coalesce but they weaken (damage) the material. This effect is encountered in brittle materials such as rocks and ceramics. The comminution of a solid body into a set of fragments by this process is sensitive to microstructural features of the body.
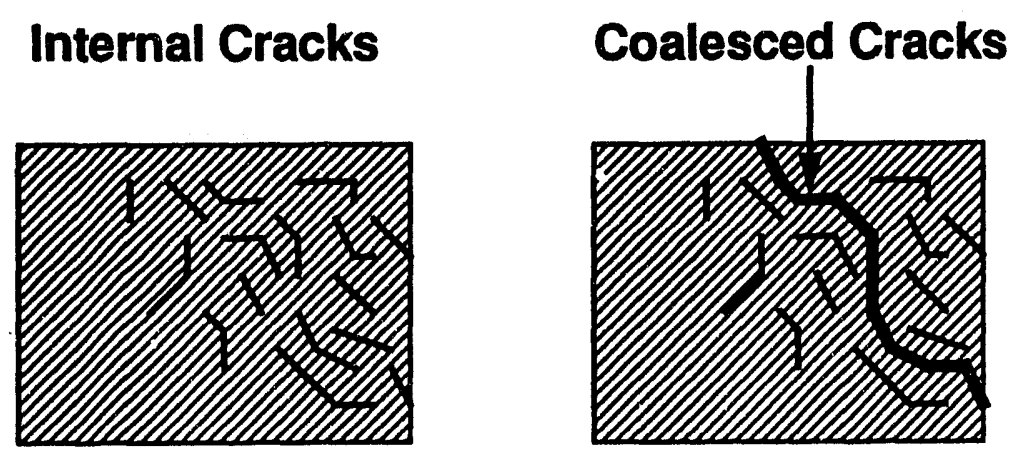

Figure 6. Formation of fractures in a shock loaded body.

Shock waves are commonly used to examine the high pressure thermodynamic states of a material. The Hugoniot, as shown in Figure 7, is the locus of thermodynamic states that may be attained by a shock wave. This locus passes through various phase boundaries related to the material. At low shock pressures the material may not be driven through the melt transition, although the pressures may be sufficient to trigger solid-solid phase transitions. As the shock pressure increases, the shock temperature increases, and the material is driven through the melt transition, then into the vapor region, and finally into the ionized region. The various phase boundaries of a material are complex functions of pressure. For example, much higher temperatures are required to melt a body when it is at a high pressure. 


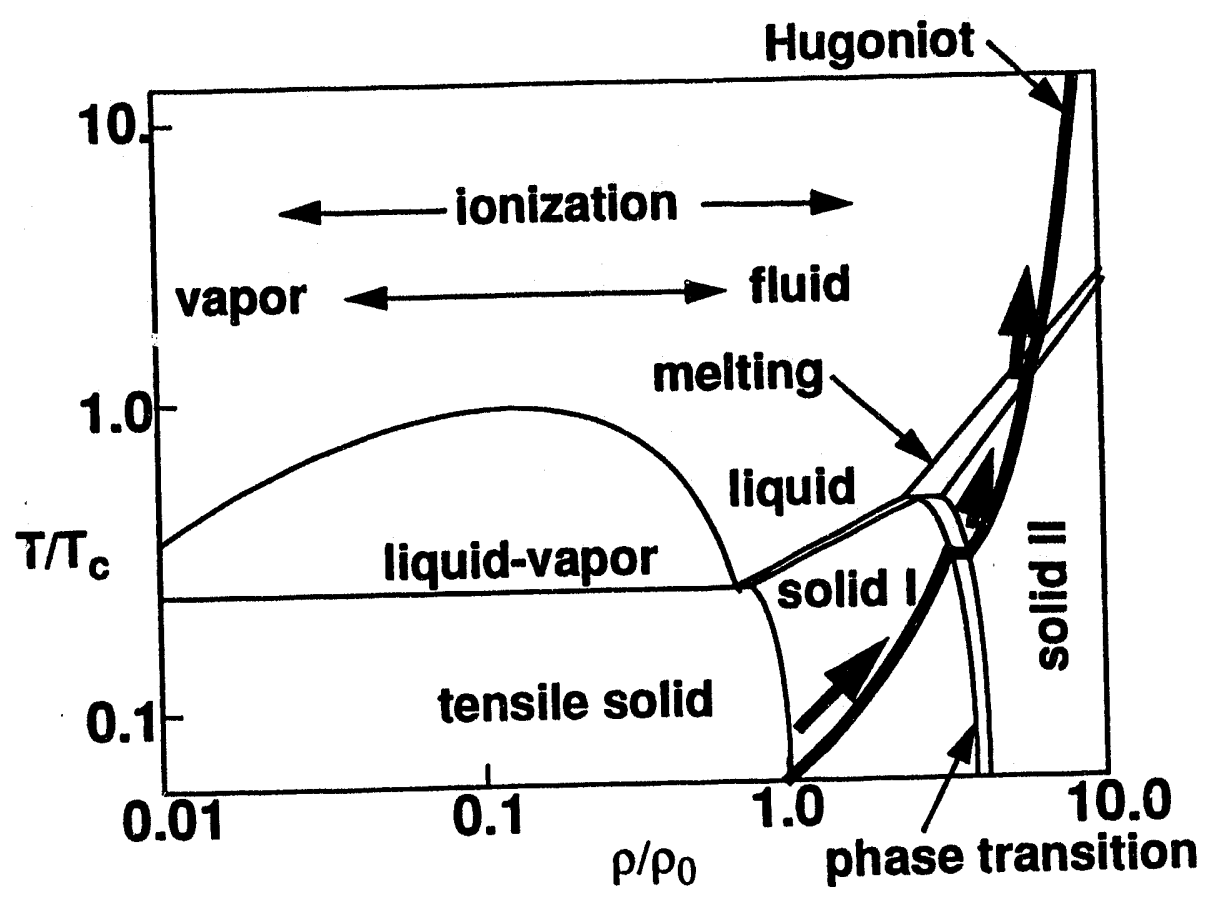

Figure 7. Plot of the shock Hugoniot in a thermodynamic plane.

The unloaded material behind a shock can be used to examine the high temperature, low density thermodynamic state of a material. Recall that a material is irreversibly heated by the passage of a shock wave, so it is hotter when it unloads. A strongly shocked material will unload 'to a hot solid, liquid, or a mixed liquid-vapor state, as shown in Figure 8. At very high shock pressures, the shocked material can completely vaporize or even ionize upon unloading. Such effects are of interest in astrophysics, for example. However, the shock waves generated by photoablation with medical lasers have pressures which are far too small for these phenomena to occur. The reader should note that in both Figures 7 and $8, T$ is the temperature, $\tau_{c}$ is the critical temperature, $\rho$ is the density, and $\rho_{0}$ is the reference density of the material. Both phase diagrams in these figures are intended to be generic. 


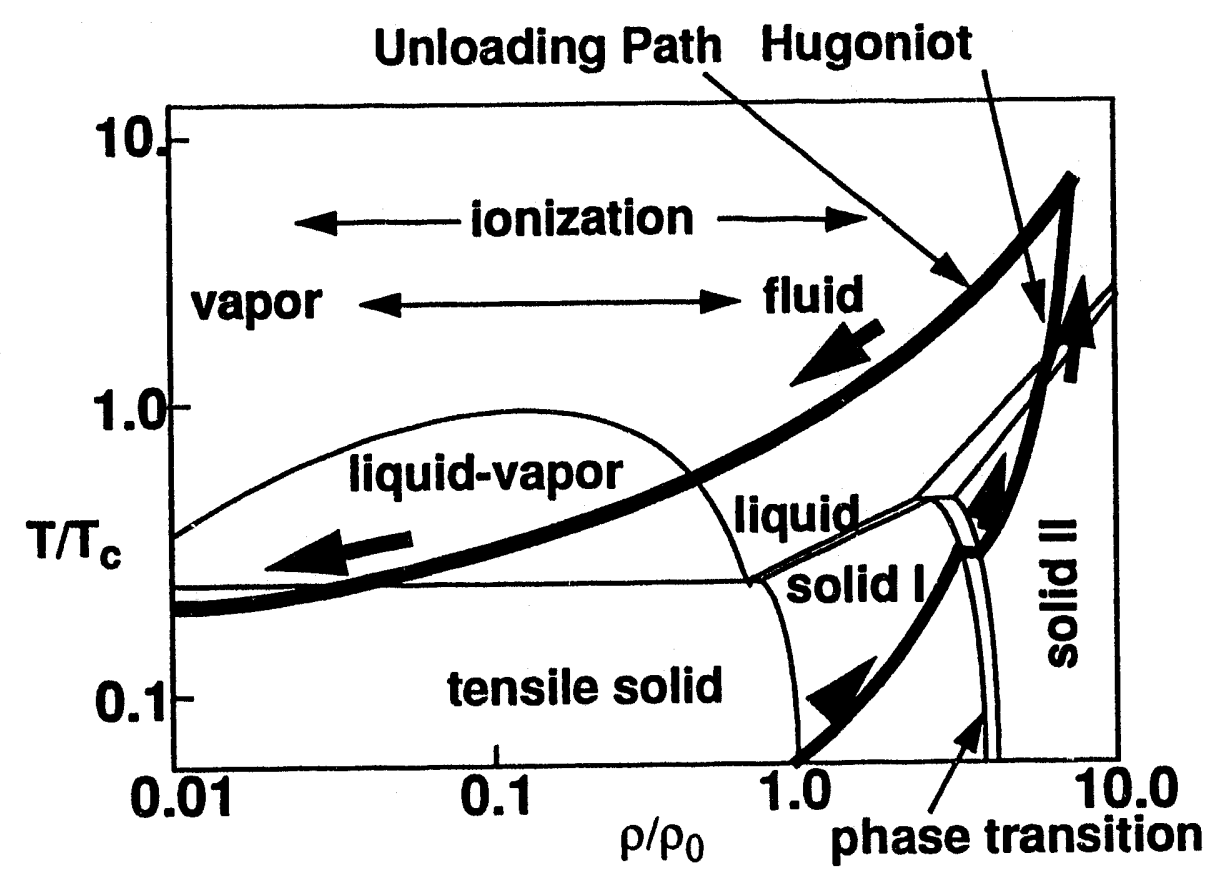

Figure 8. Plot of the release path behind a shock in a thermodynamic plane.

Many materials exhibit response which is dependent upon the rate of the loading. Most such materials appear harder as the deformation rate increases, as pictured in Figure 9. These materials are called visco-elastic or visco-plastic materials. A child's 'Silly Putty' is a good example. If you squeeze it slowly, it is soft like a piece of clay. If you throw it to the ground, it will bounce. If you cannot resist temptation and hit it with a hammer, then it will shatter like a piece of glass. Also, visco-elastic behavior is typically very sensitive to the temperature of the material.

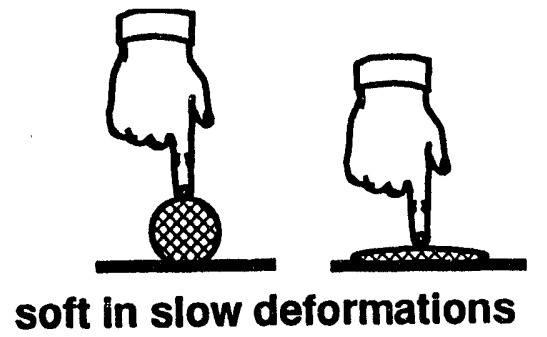

Figure 9. The behavior of visco-elastic materials. 


\subsection{Tissue Mechanical Behavior}

Tissue directly evaporates under the absorption of laser pulse energy. The equation of state of tissue enters the picture because evaporation of the tissue is a phase change that must be accurately described. It has been observed that for various purposes, water, water saturated gels, and polymeric materials are all adequate simulants for tissue when studying thermal phenomena associated with ablation. It is not at all clear to the authors what good mechanical analogs for tissue might be. This will depend upon the type of mechanical loading that the tissue is subjected to. We emphasize that in a fundamental simulation approach, the light absorption behavior of the tissue (its opacity), whether cool, evaporated, or plasma, is also linked to the equation of state. Good reviews of equations of state are Ahrens ${ }^{1}$, which emphasizes the equation of state of solid substances, and Asay and $K_{\text {Kerley }}{ }^{2}$, which also discusses the equations of state of hot matter and plasmas.

Tissue mechanical response to a laser pulse involves two physical processes: the tissue absorption of the laser pulse, with subsequent ablation, and the response of the tissue to the resulting dynamic load, typically in the form of the stress waves discussed above. While ablation is dominated by laser pulse characteristics and tissue absorptivity characteristics and equation of state summarized above, the response of the tissue to the ablation pressures is dominated by the mechanical constitutive behavior of the tissue (here we neglect long time duration thermal loads).

Immediate wave propagation phenomena associated with ablation pressures are important. These generated compressive waves are directly responsible for breakup of stones, for example. In this case, the compressional shock waves reflect from the free boundaries of stones or from internal heterogeneities, forming rarefaction waves that induce internal spall in the stone. The same types of waves can break or produce large strains in other organic structures, such as the preferential tearing observed in laser illumination of aorta. Also, shock wave propagation can independently heat tissue, and thus magnify the overall thermal damage that neighboring tissue to the interaction volume may undergo. It would be very valuable to predict the distances at which these wave disturbances have influence, and the nature of that influence, for given laser-tissue interactions.

Ablative pressures also produce longer time, large strain disturbances, as tissue responds to the pressure by moving. In confined geometry, these strains can be quite large. Fabbro, et $\mathrm{al}^{6}$ note that by far the largest percentage of impulse delivered to a target in a confined geometry by a laser pulse comes from late-time accelerations due to the ablated material. By "late-time" we mean 
times much longer (factors of ten to one hundred or more) than the length of the laser pulse. For nanosecond pulses, the ablated gases may produce a substantial deformation pressure on surrounding tissue structures for as long as one microsecond. It would be of great interest to predict with some accuracy the resulting behavior of tissue, in particular joints, bones, and other "structural" body elements, under these deformations.

For accurate simulations of laser-tissue interactions, we require accurate constitutive models for the tissue of interest. Accurate mechanical characterization of the tissue is as important as accurate absorption characterization, and just as difficult. This is a difficult subject, primarily because of the heterogeneous and non-isotropic composition of tissue. An extensive survey of current knowledge about tissue constitutive behavior is Fung ${ }^{7}$. We will simplify the present discussion and briefly consider the constitutive description of bone and skin:

- Bone is a highly anisotropic, nonlinear viscoelastic material. In certain respects, the mechanical properties of bone have the familiarity of classical engineering materials, with compressive and tensile strengths somewhat lower than seen in soft structural metals. Data for bone in compression, tension, and torsion (parallel to the bone axis) are presented. These data could be used to develop computational models for isotropic response. Anisotropic models would be considerably more difficult to develop. Bone functions normally in the human body under small strains. It's response is brittle - in other words, it fractures - under significant $\mathrm{cc}$. Apressive and bending strains. The response of bone to short, intense stress waves is not discussed in Fung's book. The anisotropy of this substance would cause anisotropic propagation of such pulses in the material, creating inhomogeneous regions of residual stress (and possibly fracture).

- Skin, blood vessels, muscle and tendon, and other soft biological solids can also be modeled as nonlinear viscoelastic materials. A variety of stress-strain data for these materials are presented in Fung's book that could aid in the development of simplified models for computational purposes. The softer tissues are functional under much larger deformations than bone. There are two major implications of bone and softer tissue being nonlinear viscoelastic materials. First, this type of constitutive behavior implies that hysteretic effects can be important for cyclic loading of tissues, such as will occur for pulsed laser surgical procedures. Second, the loading rates of tissue by photoablation may strongly change the tissue response. Recall that viscoelastic solids tend to respond more as elastic substances 
under higher loading rates, whereas they have a more fluid response for slower loading rates. For example, this may imply that the longer duration loading due to ablation gases may have a larger tendency to produce mechanical damage.

The greatest weakness of the data in Fung for our present application is that it originates in quasi-static mechanical tests. As we have emphasized, rate-dependence is very important and can qualitatively change the acquired data. Experimental programs to acquire constitutive data for tissue under dynamic loads similar to those resulting from photoablation are necessary for precision simulations.

A final consideration is the fracture behavior of tissue, and it is this area which is the most difficult to acquire experimental data. This subject is typically different than the usual constitutive theories used to describe the compressive response of materials. Perhaps the largest reason for this is the linkage of the fracture of materials to microstructural features. Continuum constitutive models attempt to avoid detailed treatment of microstructure, but it is not clear that microstructure can be avoided for fracture analysis. Since tissue has such a heterogeneous microstructure, it is likely that the microstructural details will be even more important for assessing fracture phenomena associated with laser-tissue interactions. In the context of the present discussion, this is most important for calcified plaque and body stones. Because fracture may be dominated by tissue heterogeneity coherent measurements can be very difficult. Modeling that is similar to that used for fracture in geologic media (Grady and Kipp ${ }^{9}$ and Curran, et $\mathrm{al}^{3}$ ) is probably of use for analyzing stone and plaque fracture, however, and it allows researchers to appropriately determine fracture thresholds that have meaning while still accounting for the heterogeneities which plague tissue fracture response. These approaches have been successfully integrated into the computational structure that we are describing in this paper.

\subsection{Computational aspects of material motion}

Once the absorption and mechanical response aspects of tissue have been established, the most important task is to compute the material motion and mechanical response to that motion resulting from laser pulse absorption in tissues. Computational solutions of the laws of conservation of mass, energy, and momentum lie at the heart of this treatment. These equations must be supplemented by constitutive descriptions of the tissue, in addition to the treatment of the laser pulse as an external, 
geometrically distinct energy source. Such solutions are nontrivial to perform, especially in two and three dimensions, but great advances in this field are represented by the latest generation of computational wave propagation tools.

For dynamic material response analyses, finite difference techniques, with explicit time step constraints, are common. The computational approaches typically have two broad classifications: Lagrangian, in which the computational discretization (mesh) moves with the material; and Eulerian, in which the material moves through a fixed computational grid. A third approach is likely to represent the next major generation of this type of computer program, the so-called Arbitrary Lagrangian-Eulerian (ALE) methods, which combine the strengths of both Lagrangian and Eulerian techniques. In Figure 10, we illustrate in a simplified manner differences between Lagrangian and Eulerian techniques during the laser absorption process.

The Lagrangian computational approach is characterized by a computational mesh which is fixed with the material, so that the mesh moves and deforms as the material moves. This approach is highly accurate and efficient while the mesh is reasonably uniform and undistorted. It also has the advantage that it is somewhat adaptive: the mesh refines naturally under the influence of compressive stress waves. The method's accuracy and efficiency quickly begin to degrade as the material undergoes large deformations. Material fracture can also be difficult to model in this description. Finally, the expansion of ablated material can be difficult to perform without numerical mesh tangling. The Lagrangian approach is a traditional means of simulating the small deformation response of solid bodies to dynamic loads.

In the Eulerian approach, the computational mesh is fixed in space and the material moves through the mesh. This method was historically applied to fluid motions in which large amounts of vorticity and shear were present. The method has been extended and applied to the motion of solids during the past twenty years. The disadvantage of this method is that considerably more computational work is required to maintain accuracy. It can also be difficult to model materials having the complexity of tissue. However, advantages include the ability to simulate fragmenting solids. Also, the expanded regions of the ablated material do not require special numerical treatment.

Both computational approaches have successfully integrated laser deposition with the numerical treatment of material motion. A good and up-to-date description of the numerical discretization and solution of the conservation laws in both of these descriptions is found in McGlaun and Yarrington ${ }^{12}$. We invite interested reader to read this article. 


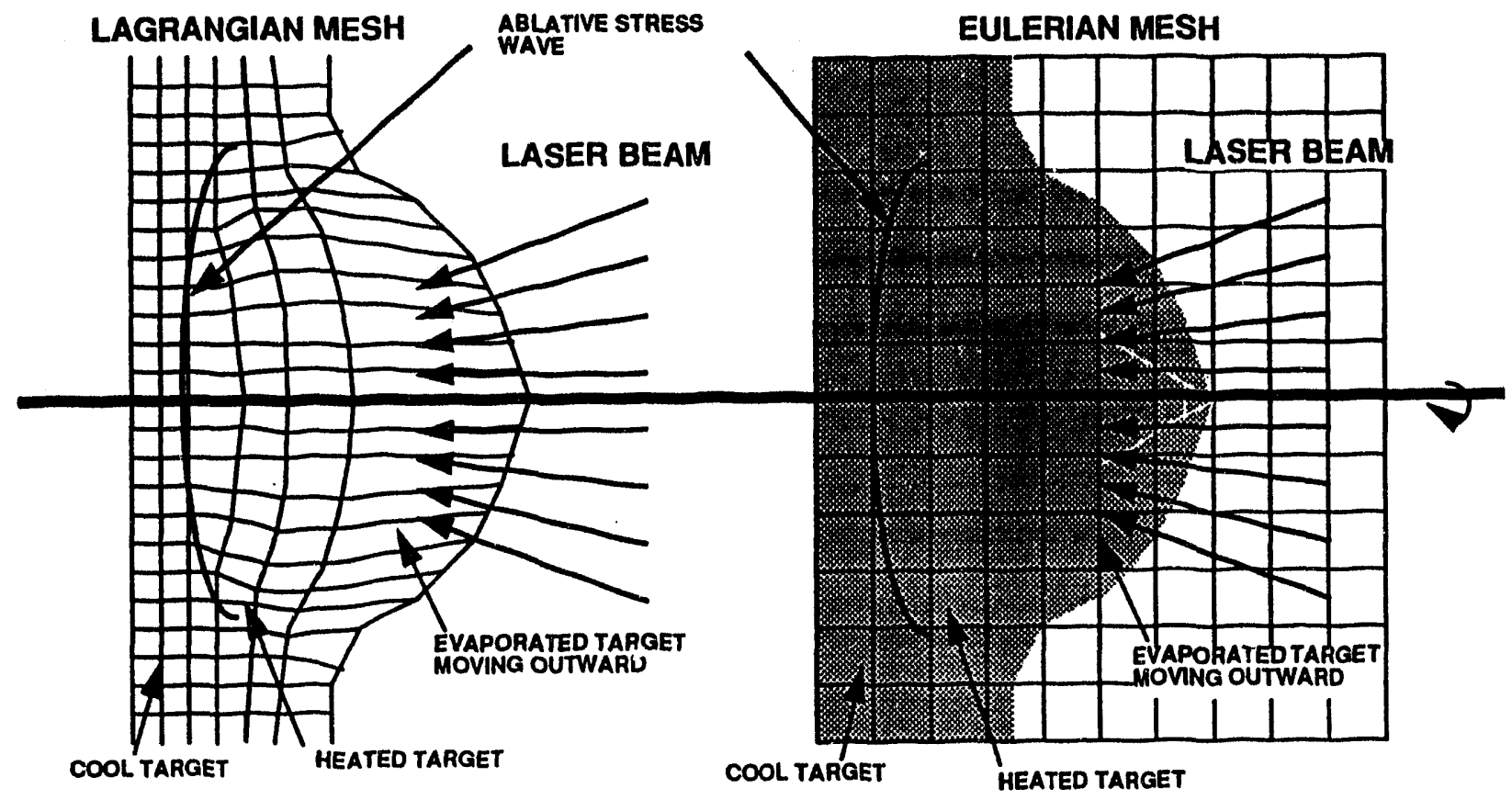

Figure 10. Lagrangian and Eulerian pictures of material motion during laser pulse absorption.

\section{CONCLUSIONS}

Computational analysis of the mechanical, as well as the thermal, effects of laser photoablation of tissue is fully feasible with current computational packages. A variety of information is required as input in order to perform accurate calculations. This information includes accurate characterization of the spatial and temporal features of the laser pulse, tissue light absorption data and thermal response data, and tissue constitutive data under non-static compressive and tensile loading conditions. From such input, very general predictions about induced tissue motion and the residual mechanical and thermal state of neighboring tissue can be made, using standard computational approaches for modeling laser light absorption and by numerically solving the conservation laws of mass, momentum, and energy. The speed and storage capacity of modern supercomputers and workstations is sufficient to perform such calculations. 
The greatest uncertainties that arise in the computational approach that we have described are related to tissue characteristics. Tissue is a complex substance. A large amount of information is available related to its thermal and mechanical properties, but it is not clear that all of the information that is needed for large-scale computations is available. Also, the data have intrinsic uncertainties. In the case of mechanical data, there is a lack of constitutive data for the response of tissue to dynamic loads similar to those induced by laser photoablation. These uncertainties will have to be resolved before we can have strong confidence in computational simulations. Nonetheless, great benefits can be gained from computational analyses of the type that we have proposed. These include fuller understanding of in vitro and in vivo experimental data, the ability to supplement costly and difficult laboratory procedures, and the possibility of refining proposed experimental and clinical techniques prior to their actual implementation.

\section{BIBLIOGRAPHY}

1. T. J. Ahrens, "Equation of State," In: High-Pressure Shock Compression of Solids, ed. J. R. Asay and M. Shahinpoor, Springer, New York, pp. 75-113, 1993.

2. J. R. Asay and G. I. Kerley, “The Response of Materials to Dynamic Loading," Int. J. Impact Engng, Vol. 5, pp. 69-99, 1987.

3. D. R. Curran, L. Seaman, and D. M. Shockey, "Dynamic Failure of Solids," Phys. Rep., Vol. 147, pp. 253-388, 1987.

4. R. S. Dingus and B. P. Shafer, "Laser-Induced Shock Wave Effects in Materials," In: Laser-Tissue Interaction, SPIE Vol. 1202, pp. 36-45, 1990.

5. K. Dörschel and G. Müller, "Dosimetry for Photoablation Technique," SPIE Vol. IS5, pp. 226-237, 1989.

6. R. Fabbro, J. Fournier, P. Ballard, D. Devaux, and J. Virmont, "Physical Study of Laser-Produced Plasma in Confined Geometry," J. Appl. Phys.; Vol. 68; No. 2; 1990; 775-784

7. Y. C. Fung, Biomechanics, Mechanical Properties of Living Tissues, 2nd Edition, Springer, New York, 1993.

8. N. P. Furzikov, "Different Lasers for Angioplasty: Thermooptical Comparison," IEEE J. Quant. Electron., Vol. 23, pp. 1751-1755, 1987. 
9. D. E. Grady and M. E. Kipp, "Fragmentation of Solids Under Dynamic Loading," In: Structural Failure, John Wiley \& Sons, New York, pp. 1-40, 1989.

10. V. I. Konov, A. M. Prokhorov, and I. A. Shcherbakov, "Perspectives of Powerful Laser Technique for Medicine," In: Euture Trends in Biomedical Applications of Lasers, SPIE Vol. 1525, pp. 250-252, 1991.

11. W. L. Kruer, The Physics of Laser Plasma Interactions, Addison-Wesley, New York, 1988.

12. J. M. McGlaun and P. Yarrington, "Large Deformation Wave Codes," In: High-Pressure Shock Compression of Solids, ed. J. R. Asay and M. Shahinpoor, Springer, New York, pp. 323-353, 1993.

13. G. Müller, K. Dörschel, H. Kar, "The Tissue Photoablation Process with short pulsed Lasers," In: Biooptics: Oppics in Biomedicine and Environmental Sciences, SPIE Vol. 1524, pp. 150-177, 1991.

14. N. S. Nishioka and R. R. Anderson, "Fragmentation of Biliary Calculi With Tunable Dye Lasers," Gastrointest. Endosc., Vol. 32,p. 157, 1986.

15. A. E. Profio, "Light Transport in Tissue", Applied Optics, Vol. 28, pp. 2216-2222, 1989.

16. G. Rinker and G. Bohannon, "Focused Beam Ray Tracing for Laser Fusion," IEEE Trans. Plasma Scienced, Vol. 8, pp. $55-60,1980$.

17. A. Sagi, A. Shitzer, A. Avidor, A. Akselrod, A. Katsir, "Heating and Cutting of Biological Tissues by Highly Absorbed Laser Beams: Theoretical Model and Experiments," In: Laser-Tissue Interaction, SPIE Vol. 1202, pp. 228-233, 1990.

18. D. L. Singleton, G. Paraskevopoulos, R. S. Taylor, and L. A. J. Higginson, "Excimer Laser Angioplasty: Tissue Ablation, Arterial Response, and Fiber Optic Delivery," IEEE J. Quant. Electron., Vol. 23, 1772-1782, 1987.

19. D. Sliney and M. Wolbarsht, Safety With Lasers and OTher Optical Sources, Plenum Press, New York, 1980.

20. A. Sona, "Lasers for Biooptics," In: Biooptics: Optics in Biomedicine and Environmental Sciences, SPIE Vol. 1524, pp. 2-16,1991. 
21. R. Srinivasan, K. G. Casey, and J. D. Haller, "Subnanosecond Probing of the Ablation of Soft Plaque From Arterial Wall by $308 \mathrm{~nm}$ Laser Pulses Delivered Through a Fiber," IEEE J. Quant. Electron., pp. 2279-2283, 1990.

22. P. Teng, N. S. Nishioka, R. F. Anderson, and T. F. Deutsch, "Acoustic Studies of the Role of Immersion in PlasmaMediated Laser Ablation," IEEE J. Quant. Electron, Vol. 23, pp. 1845-1852, 1987.

23. J. T. Walsh and T. F. Deutsch, "Er.Y AG Laser Ablation of Tissue: Measurement of Ablation Rates," Laser N. g. Med., Vol. 9, pp. 327-337, 1989.

24. J. T. Walsh, T. J. Flotte, and T. F. Deutsch, "Er:YAG Laser Ablation of Tissue: Effect $0_{2}$, se Duration and Tissue Type on Thermal Damage," Lasers Surg. Med., Vol. 9, pp. 314-326, 1989.

25. A. J. Welch, J. W. Valvano, J. A. Pearce, L. J. Hayes, and M. Motamedi, "Effect of Laser Radiation on Tissue During Laser Angioplasty," Lasers Surg. Med., Vol. 5, pp. 251-264, 1985.

26. A. D. Zweig and H. P. Weber, "Mechanical and Thermal Parameters in Pulsed Laser Cutting of Tissue," IEEE J. Quant. Electron, Vol. 23, pp. 1787-1793, 1987.

\section{ACKNOWLEDGEMENT}

This work performed at Sandia National Laboratories supported by the U. S. Department of Energy under contract number DEAC04-94AL85000. 

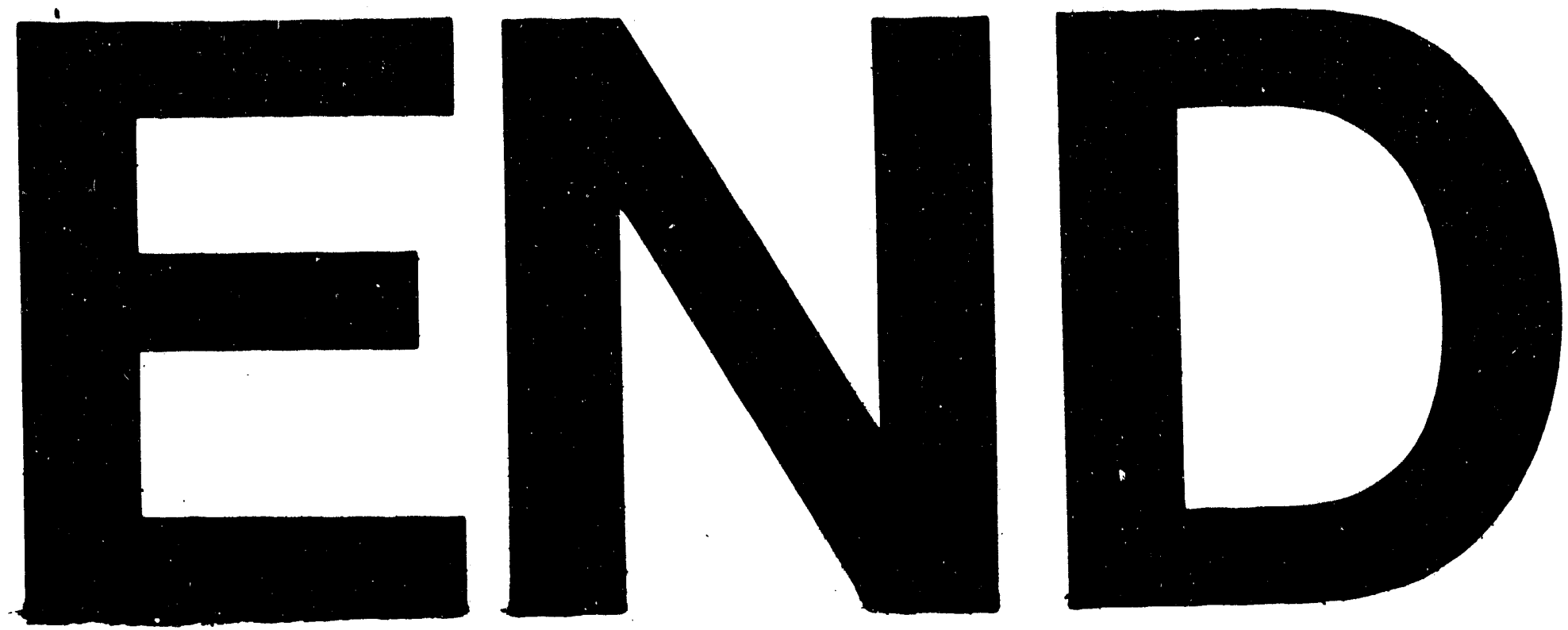

$\infty$
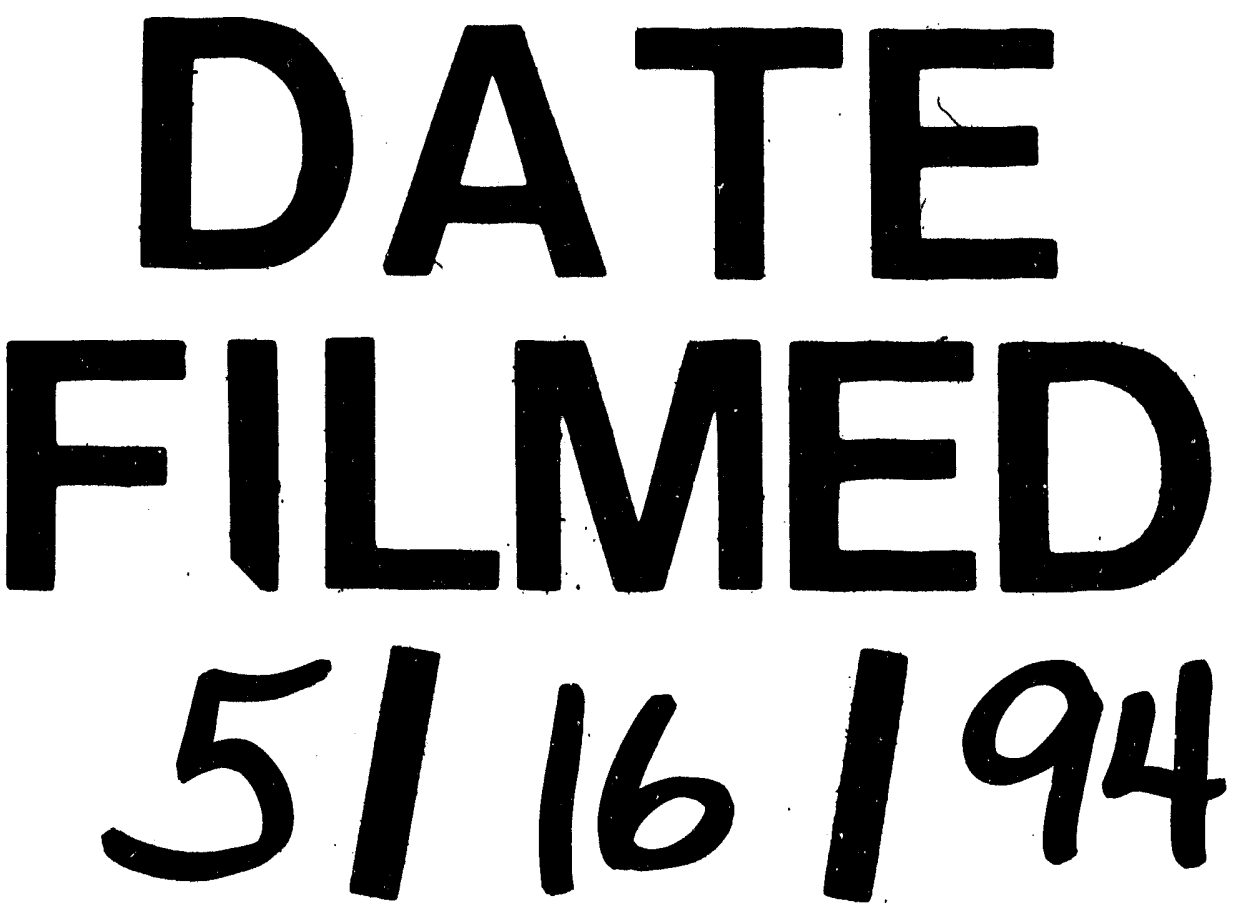


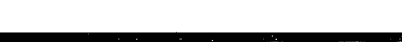<smiles>CC</smiles>
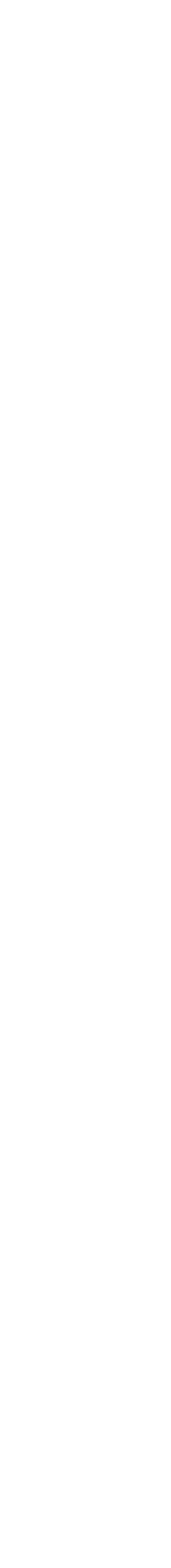